\title{
CLCN7 and TCIRG1 mutations in a single family: Evidence for digenic inheritance of osteopetrosis
}

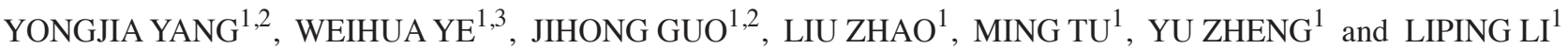 \\ ${ }^{1}$ The Laboratory of Genetics and Metabolism, Hunan Children's Research Institute (HCRI), Hunan Children's Hospital, \\ University of South China, Changsha, Hunan 410007; ${ }^{2}$ State Key Laboratory of Medical Genetics, Central South University, \\ Changsha, Hunan 410008; ${ }^{3}$ The Department of Orthopedics, Hunan Children's Research Institute (HCRI), \\ Hunan Children's Hospital, University of South China, Changsha, Hunan 410007, P.R. China
}

Received March 9, 2018; Accepted October 22, 2018

DOI: $10.3892 / \mathrm{mmr} .2018 .9648$

\begin{abstract}
Osteopetrosis is a monogenic condition with various inheritance patterns, including autosomal dominant, autosomal recessive and X-linked. Several disease-causing genes have been identified and three distinguished types of osteopetrosis have been reported. In the present study, a family with osteopetrosis was investigated. Two novel mutations in chloride voltage-gated channel 7 (CLCN7) and T cell immune regulator 1 (TCIRG1) were identified by exome sequencing, Sanger sequencing and microsatellite marker analysis. The CLCN7 mutation occurred in amino acid R286, the same position as previously reported. The TCIRG1 mutation occurred on a splicing site of exon 15, thereby leading to a truncated transcript. These two mutations were undetected in 496 ethnic-matched controls. To the best of our knowledge, this is the first report of human osteopetrosis involving digenic inheritance in a single family, which has important implications for decisions on clinical therapeutic regimen, prognosis evaluation and antenatal diagnosis.
\end{abstract}

\section{Introduction}

Osteopetrosis is a rare, heritable bone disease that is characterized by increased bone density due to failure of bone resorption by osteoclasts (1). Osteopetrosis is also known as marble bone or Albers-Schönberg disease. This disease was first reported in 1904 by the German radiologist Heinrich Albers-Schönberg (1,2). Osteopetrosis is currently considered a monogenic disease and is transmitted through various inheritance patterns, including autosomal recessive, autosomal

Correspondence to: Dr Yu Zheng or Dr Liping Li, The Laboratory of Genetics and Metabolism, Hunan Children's Research Institute (HCRI), Hunan Children's Hospital, University of South China, 86 Ziyuan Road, Changsha, Hunan 410007, P.R. China

E-mail: zhengyu@sklmg.edu.cn

E-mail: 13974871993@163.com

Key words: digenic inheritance, osteopetrosis, CLCN7, TCIRG1 dominant or X-linked (2-4). On the basis of the aforementioned inheritance patterns, researchers have described several distinct types of osteopetrosis, including infantile malignant autosomal recessive osteopetrosis (ARO), intermediate osteopetrosis (IO), which can be either recessive or dominant, and autosomal dominant osteopetrosis (ADO) $(1,2,5)$.

ARO is the most severe form of the disease, whereas ADO is considered the mildest form. Individuals with ADO usually exhibit mild symptoms, including high bone density, increased bone fracture, scoliosis, arthritis and osteomyelitis. These phenotypes become evident during late childhood or adulthood $(1,5,6)$. ADO can be further subdivided into two forms, including ADO-I, which is caused by a low-density lipoprotein receptor-related protein 5 mutation (7) and ADO-II, which is caused by a chloride voltage-gated channel 7 (CLCN7) mutation (3). Individuals with ARO exhibit apparent phenotypes in early infancy, including frequent bone fracture, compression of cranial nerves and bone marrow failure, thereby leading to severe anemia and life-threatening events, hepatosplenomegaly, brain abnormalities, epilepsy and developmental delay $(1,5,8)$. IAO is an intermediate form of osteopetrosis. Several cases of IAO have been reported (9) and IAO can be transmitted through autosomal dominant and autosomal recessive inheritance $(2,9,10)$. The symptoms for IAO that occur during childhood include risk of fracture, mild anemia, renal tubular acidosis and calcification $(9,10)$.

At least 10 disease-causing genes have been identified to date, which account for $70 \%$ of total osteopetrosis cases $(1,2)$. Among these genes, CLCN7 and T cell immune regulator 1 (TCIRG1) are the most frequently mutated genes in human osteopetrosis (11). Monogenic mutations of CLCN7 can cause recessive IAO (10), ADO-II (12) and ARO (13), whereas monogenic mutations of TCIRG1 can cause recessive IAO (4) and ARO (14).

In the present study, exome sequencing, Sanger sequencing and microsatellite marker mapping were performed on a family with ADO-II. The results revealed two novel heterozygous mutations in CLCN7 and TCIRG1. These data indicated the digenic inheritance of osteopetrosis and highlighted the importance of next-generation sequencing to diagnose human osteopetrosis. 


\section{Materials and methods}

Ethics statement. The present study was approved by the Ethics Committee of The Hunan Children's Hospital (Changsha, China). The study was carried out according to the principles of the 2008 Edition of the Declaration of Helsinki. Prior to participating in the study, written informed consent was obtained from all subjects or guardians of children.

Study subjects. A Han Chinese family residing in the rural areas of Hunan province with osteopetrosis was recruited for this study (Fig. 1). In addition, 496 ethnic- and region-matched controls were included. These controls were selected from patients (10-16 years old, 263 males and 233 females) who came to Hunan Children's Hospital for GTG banding between June 2016 and July 2017; individuals with skeletal disorders were excluded. The family comprised five individuals, including two affected siblings (II:2 and II:3), an unaffected mother (I:2) and an unaffected sibling (II:1; Fig. 1). The father (I:1) did not participate in the study. For each participant, genomic DNA was extracted from peripheral blood (2-5 $\mathrm{ml}$ in sodium heparin tubes) using a DNA isolation kit (cat. no. D3392-02; Omega Bio-Tek, Inc., Norcross, GA, USA) according to the manufacturer's instructions.

Whole exome sequencing. Briefly, $3 \mu \mathrm{g}$ genomic DNA from II:2 and II:3 was fragmented with a bath sonicator (duty cycle, $10 \%$; intensity, 5 ; cycles per burst, $200 ; 3 \min$ for $25^{\circ} \mathrm{C}$; Covaris, Inc., Woburn, MA, USA) and prepared following Illumina Genomic DNA Sample Prep kit (cat. no. FC-102-1001; Illumina, Inc., San Diego, CA, USA) according to the manufacturer's protocol, to generate adapter-ligated DNA library. The prepared DNA was amplified using ligation-mediated polymerase chain reaction. Phusion Master Mix. The following thermocycling conditions were used: $98^{\circ} \mathrm{C}$ for $30 \mathrm{sec}$, followed by 12 cycles of $98^{\circ} \mathrm{C}$ for $10 \mathrm{sec}, 65^{\circ} \mathrm{C}$ for $30 \mathrm{sec}$ and $72^{\circ} \mathrm{C}$ for $30 \mathrm{sec}$; and $72^{\circ} \mathrm{C}$ for $5 \mathrm{~min}$. Next, DNA was captured using NimbleGen 2.1M HD array (Roche Applied Science, Madison, WI, USA) according to the manufacturer's protocol. The captured library was sequenced on a HiSeq ${ }^{\mathrm{TM}} 2000$ Sequencing system $(2 \times 90$ bp end reads; Illumina, Inc.) and the Illumina base calling softwareCASAVA (version 1.7) was used to convert raw bcf files to fastq file with default parameters. After trimming the low quality bases (Phred score <20), the obtained clean reads were mapped to the reference human genome (hg19; genome.ucsc.edu) using the Burrows-Wheeler Aligner (version 0.7.2; bio-bwa.sourceforge.net) with up to two mismatches. The alignment files (.bam) were generated using SAMtools (samtools.sourceforge.net) and reads with low mapping quality $(<Q 30)$ were filtered out. Duplicate reads that may be derived from PCR artifacts were removed with Picard (broadinstitute.github.io/picard/) using default parameters. Short read alignment and annotation visualization were performed using the Integrative Genomics Viewer (software. broadinstitute.org/software/igv/). The percentage alignment of the clean reads to the exome regions was obtained using custom Perl scripts on the basis of the alignment files. Single nucleotide variants (SNVs), as well as short insertions and deletions (indels), were detected by Genome Analysis
Toolkit (software.broadinstitute.org/gatk/). Comprehensive annotations of all of the detected SNVs and indels were performed by ANNOVAR (annovar.openbioinformatics. org/en/latest/). The annotations included function implication (gene region, functional effect, mRNA GenBank accession number, amino acid change, cytoband etc.), and allele frequencies in dbSNP (ncbi.nlm.nih.gov/projects/SNP/), 1000 Genomes (1000genomes.org), ESP6500 (evs.gs.washington. edu/EVS/) and ExAc (exac.broadinstitute.org/). Damaging missense mutations were predicted by SIFT (sift.bii.a-star. edu.sg/), PolyPhen-2 (genetics.bwh.harvard.edu/pph2/) and MutationTaster (mutationtaster.org/).

Sanger sequencing. To validate the possible disease-causing variants, family gDNA underwent PCR using the Goldstar ${ }^{\circledR}$ PCR kit (cat. no. CW0655M; Jiangsu Kangwei Century Biotechnology Co., Ltd., Beijing, China) and Sanger sequencing. The following primers were used: $C L C N 7$, forward 5'-GACTCGGTTGTCCTGAAAGC-3', reverse 5'-GGC TGGACCCAAGTACACTG-3' (predicted size, $371 \mathrm{bp}$ ); and TCIRG1, forward, 5'-CAGTGAGCACACCTCCCTCT-3', and reverse, 5'-GTGAGGGTCTGCCTCCATAA-3' (predicted size, $472 \mathrm{bp}$ ). The following PCR conditions were used: $94^{\circ} \mathrm{C}$ for $5 \mathrm{~min}$; followed by 35 cycles of $94^{\circ} \mathrm{C}$ for $30 \mathrm{sec}, 60^{\circ} \mathrm{C}$ for $30 \mathrm{sec}$ and $72^{\circ} \mathrm{C}$ for $40 \mathrm{sec}$; and a final extension at $72^{\circ} \mathrm{C}$ for 5 min. The BigDye ${ }^{\circledR}$ Terminator v3.1 Cycle Sequencing kit (Applied Biosystems; Thermo Fisher Scientific, Inc., Waltham, MA, USA) was used for the Sanger sequencing according to manufacturer's protocol. The amplified PCR products were purified by $70 \%$ ethanol and then were run on an Applied Biosystems $^{\mathrm{TM}} 3500$ Series Genetic Analyzer (Applied Biosystems).

Microsatellite marker analysis. Five informative microsatellite markers, namely D16S521, D16S3027, D16S423, D11S987 and D11S4162, were used for segregation analysis. This genotyping method was described previously (15). A total of $0.8 \mu 1$ PCR products, $0.2 \mu 1 \mathrm{GeneScan}^{\mathrm{TM}} 500 \mathrm{LIZ}^{\mathrm{TM}}$ dye Size Standard (Thermo Fisher Scientific, Inc.) and $9 \mu \mathrm{l} \mathrm{Hi-Di}$ Formanmide (Thermo Fisher Scientific, Inc.) were detected on an Applied Biosystems ${ }^{\text {TM }} 3500$ Series Genetic Analyzer (Applied Biosystems). Cyrillic program (version 2; www. apbenson.com/cyrillic-downloads) was used for constructing haplotypes.

Validation of TCIRG1 splicing mutation on the mRNA level. Total RNA was extracted from peripheral blood mononuclear cells obtained from patient II:3 and control I:2 using the RNAiso Plus kit (Takara Biotechnology Co., Ltd., Dalian, China). cDNA was synthesized from $1 \mu \mathrm{g}$ RNA using PrimeScript ${ }^{\mathrm{TM}}$ II 1st Strand cDNA Synthesis kit (Takara Biotechnology Co., Ltd.) according to the manufacturer's protocol. PCR was performed using cDNA as a template with primers specific for exons 13-14, forward, 5'-CTATTTGGA GCCTGGCTGCC-3' and exon 15, reverse, 5'-CGTCAGGCA GGTCCAGCAACC-3' (predicted size, 497 bp). The amplified PCR products were run on a $1 \%$ agarose gel $(100 \mathrm{~V} ; 40 \mathrm{~min})$. The forward primer was used as the sequencing primer. The relevant PCR and Sanger sequencing conditions are the same as those mentioned above. 
Table I. Laboratory test results for II:2. ${ }^{a}$

\begin{tabular}{lrc}
\hline Variable & II:2 & Normal range \\
\hline Red-cell count $\left(10^{12}\right) / 1$ & $4.05(\downarrow)$ & $4.09-5.74$ \\
Hemoglobin $(\mathrm{g} / \mathrm{l})$ & $115(\downarrow)$ & $120-140$ \\
Hematocrit $(\%)$ & $0.334(\downarrow)$ & $0.38-0.51$ \\
Mean corpuscular volume (fl/cell) & $82.5(\downarrow)$ & $86-100$ \\
\hline
\end{tabular}

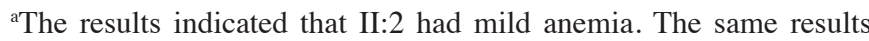
were observed for three tests taken at $\sim 30$-day intervals.

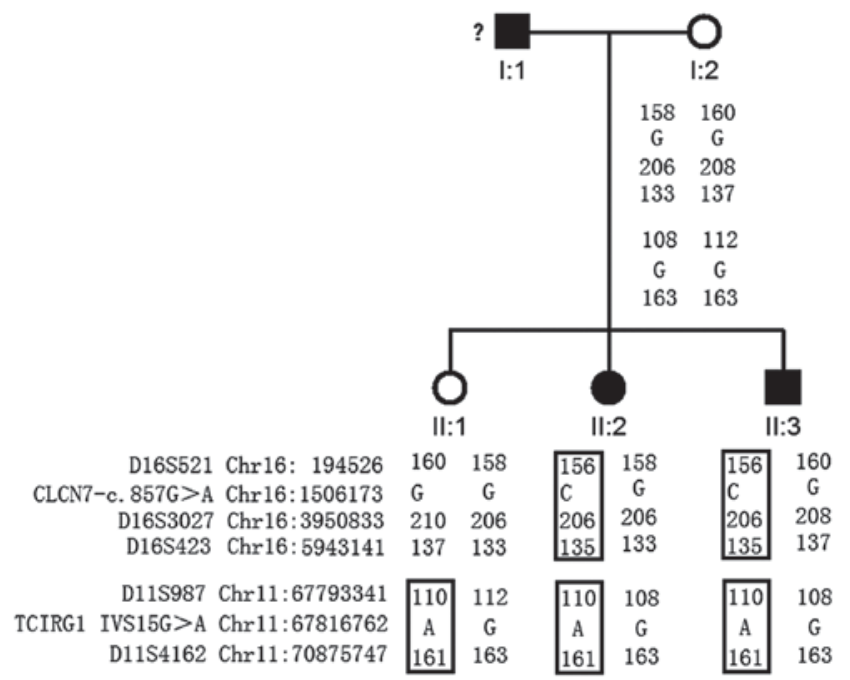

Figure 1. Pedigree of a family with osteopetrosis. The affected individuals are denoted by solid symbols. The haplotypes in the critical region on chromosomes are listed below each individual and the squares indicate where microsatellite marker analysis was performed. The status of I:1 is unknown because this individual did not agree to participate in this study.

\section{Results}

Clinical data. The X-ray results revealed that the two patients, II:2 and II:3, exhibited 'sandwich vertebrae' and 'bone-within-bone' appearance (Figs. 2 and 3). II:2 had two fractures, whereas II:3 had no fractures. The laboratory test results revealed that II:3 exhibited mild anemia (Table I). The symptoms of II: 2 and II:3 were consistent with those of ADO-II. The father, I:1, did not participate in the study; however, as described by I:2, I:1 had two fractures during childhood. Therefore, I:1 was likely also a patient with osteopetrosis.

Genetic findings. Whole exome sequencing was successfully performed on II:2 and II:3. Firstly, common variants present in public and in-house databases were filtered out. Given that II:2 and II:3 exhibited 'bone-within-bone' phenotypes, genes involved in osteopetrosis were investigated (3). Two heterozygous variants, chr11-67816762 G>A and chr16-1506173 G>C, were detected in these two patients among 10 osteopetrosis genes (data not shown). The Sanger sequencing results confirmed the two mutations (Fig. 4).

Variant chr11-67816762 G>A (IVS15+1G>A) occurred on intron 15 (the splicing site of exon 15) in TCIRG1 (NCBI reference sequence: NM_006019). In silico analysis (Mutation taster, http://www.mutationtaster.org/) predicted that the splice acceptor site was lost. Total RNA was extracted from whole blood, cDNA was synthesized and amplification was performed with the primers flanking exon 15 of TCIRG1. For patient II:3, two bands at 497 and 283 bp, were visualized on agarose gel. For control I:2, only one band was detected at 497 bp. Skipping of the whole exon 15 was confirmed by PCR and validated by Sanger sequencing (Fig. 5). Accordingly, the mutated transcript led to a frameshift mutation from the amino acid V558, which truncated the mutated protein at amino acid L614. Variant chr16-1506173 G>C was a c.857G $>C$ missense mutation in exon 10 of CLCN7 (NCBI reference sequence: NM_001287; Fig. 4). The c.857G $>$ C missense mutation changed codon 286 from CGG to CCG. This resulted in the substitution of arginine by proline (p.R286P). The R286 position was highly conserved (Fig. 6). Two known CLCN7-disease mutations on the R286 position, p.R286W and p.R286Q, have previously been reported (Table II).

Sanger sequencing was conducted to analyze the distribution of the two mutations in the family. I: 2 carried none of these mutations, II:1 only carried the TCIRG1 (IVS15+1 G>A) mutation, and II:2 and II:3 carried both mutations (Fig. 1). Furthermore, the two mutations, TCIRG1-IVS15+1G $>\mathrm{A}$ and CLCN7-c.857G >A, were undetected in the 496 region and ethnic-matched controls (data not shown).

Microsatellite marker analysis was performed in the family, of which five markers were informative. The haplotype analysis results confirmed that the two mutations were not transmitted from the unaffected mother (Fig. 1).

\section{Discussion}

Monogenic mutations of CLCN7 and TCIRG1 have previously been fully documented as the cause of human osteopetrosis (2); however, osteopetrosis is a heterogeneous disease (16). Human osteopetrosis exhibits high phenotypic and genotypic diversity $(3,17,18)$; for example, individuals with a $C L C N 7$ mutation can be asymptomatic or symptomatic, with symptoms ranging from very mild to severe $(3,17,18)$. This is consistent with the fact that the penetrance of human osteopetrosis is not complete (19). Although several studies have suggested the presence of modifier genes for human osteopetrosis, including a modifier gene on chromosome $9 q 21-9 q 22(16,20)$, the precise mechanism of the phenotypic diversities of human osteopetrosis requires further investigation.

To the best of our knowledge, the present study was the first to describe two heterogeneous mutations occurring in CLCN7 and TCIRG1 in a single family with osteopetrosis. It has been proposed that both mutations are disease-causing mutations due to the following characteristics: i) The TCIRGI mutation (IVS15+1G>A) was a novel splicing mutation, which has not been previously reported, and the splice acceptor site was lost as predicted by MutationTaster. In addition, detection of mRNA expression indicated that the whole exon 15 of TCIRG1 was skipped, and the predicted protein was truncated. ii) The CLCN7 mutation (p.R286P) was also a novel missense mutation, and MutationTaster also predicted that CLCN7 was a disease-causing mutation. In addition, the R286 position of 
Table II. Genotype and phenotype of several reported cases of osteopetrosis.

\begin{tabular}{lllc}
\hline Studies & \multicolumn{1}{c}{ Mutation } & Phenotype & (Refs.) \\
\hline Cleiren et al (2001) & CLCN7-p.R286W & ADO-II & $(12)$ \\
Frattini et al (2003) & CLCN7-p.R286Q & ADO-II & $(21)$ \\
Present study & CLCN7-p.R286P; TCIRG1-IVS 15+1G>A & ADO-II & Present study \\
Yu et al (2014) & CLCN7-IVS20-1G>T; TCIRG1-Y303X; TCIRG1-R670X & ARO & $(22)$ \\
\hline
\end{tabular}

ADO, autosomal dominant osteopetrosis; ARO, infantile autosomal malignant recessive osteopetrosis; CLCN7, chloride voltage-gated channel 7; TCIRG1, T cell immune regulator 1.
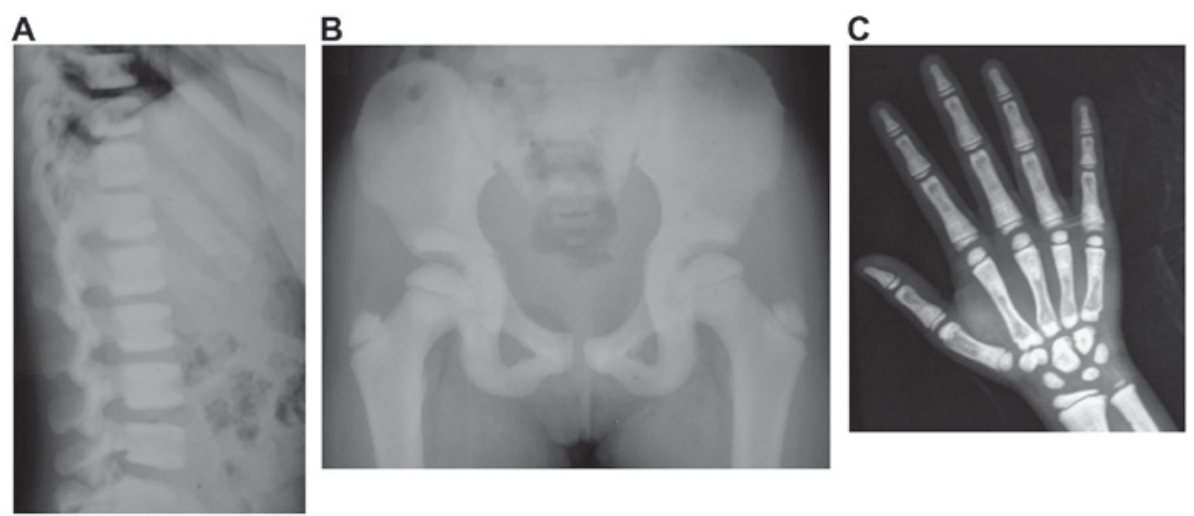

Figure 2. X-ray images of II:2 at 10.1 years old. Increased bone density or 'bone-within-bone' appearance in the (A) vertebral bodies, (B) pelvis and (C) phalanges.
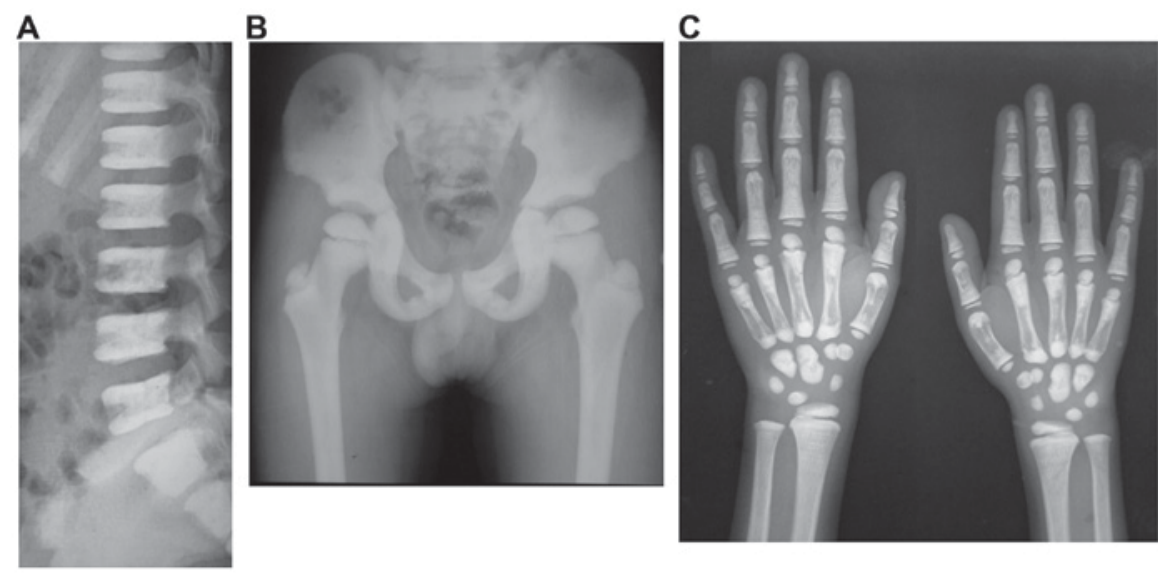

Figure 3. X-ray images of II:3 at 8.5 years old. Increased bone density or 'bone-within-bone' appearance in the (A) vertebral bodies, (B) pelvis and (C) bilateral phalanges.

CLCN7 was highly conserved among various species and two known disease mutations on the R286 position, p.R286W and p.R286Q, have previously been reported $(14,21)$.

The present study proposed the possibility of digenic inheritance of osteopetrosis, where CLCN7-p.R286P combined with TCIRG1-IVS $15+1 \mathrm{G}>\mathrm{A}$ cause ADO-II. This proposition is supported by a previous study; Yu et al (22) described an ARO case with three mutations, including two compound heterozygous mutations in TCIRG1, p.Y303X and p.R670X, and one splicing mutation in CLCN7 (IVS 20-1G>T). For the ARO case (22), TCIRG1-p.Y303X and CLCN7 IVS 20-1G>T mutations were passed on from the asymptomatic mother of the patient, and the TCIRG1-p.R670X mutation was transmitted from the asymptomatic father of the patient. In addition, the compound heterozygous nonsense mutations of TCIRG1 were sufficient to cause ARO; however, it may not be a coincidence to have mutations on two rare pathogenic genes. This condition may be due to the fact that the mutations of TCIRG1 and $C L C N 7$ interacting with each other in the two mutations exert a digenic inheritance (22).

In conclusion, the present study identified two novel gene mutations in TCIRG1 and CLCN7 in a single family, 

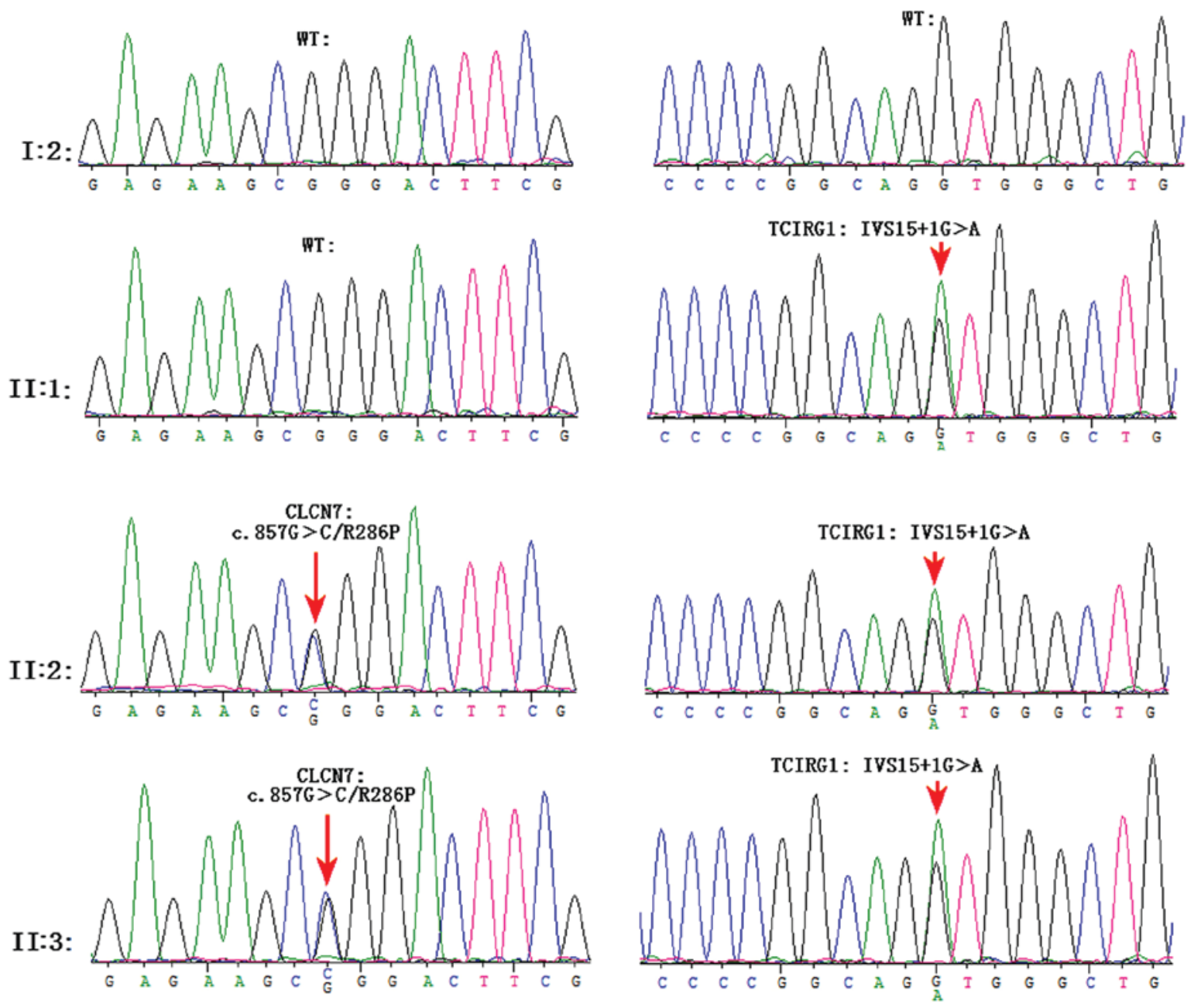

Figure 4. Sanger sequencing identified two novel mutations in the osteopetrosis family. -II:2 and II:3 carried TCIRG1-IVS15+1G>A and CLCN7-R286P mutations. II:1 carried the TCIRG1-IVS15+G>A mutation. I:2 did not carry any of these mutations. CLCN7, chloride voltage-gated channel 7; TCIRG1, T cell immune regulator 1 ; WT, wild type.

\section{Normal:}

Exon:

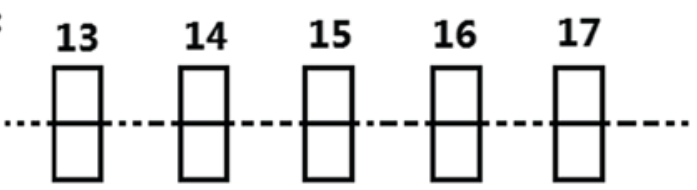

IVS $15+1 G>A$ :

Exon:
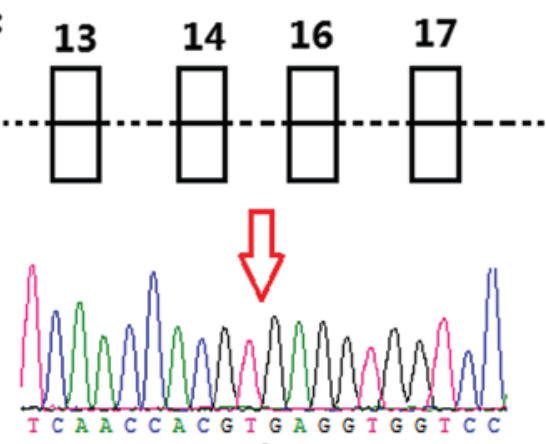

Exon 14 | Exon 16

Figure 5. Detection of the TCIRG1-IVS15+1G splicing mutation. Sanger sequencing detected that the $197 \mathrm{bp}$ fragment lacked the whole exon 15 of the TCIRG1 gene. TCIRG1, T cell immune regulator 1.

\begin{tabular}{|c|c|c|c|}
\hline & & & \\
\hline 4. sapiens & & IFEYFRRD & \\
\hline ed & & D T E K $\mid \mathrm{P}$ & \\
\hline troglodytes & 286 & D T E K $\mid R$ & DF V S \\
\hline & & $\mathrm{EK} / \mathrm{R}$ & DF I \\
\hline & & \begin{tabular}{l|l}
$\mathrm{E} K$ & $\mathrm{R}$
\end{tabular} & \\
\hline & 284 & D T E K $\mid R$ & DF V S \\
\hline & 282 & I E K $\mid R$ & DF \\
\hline & 275 & T E K $/ R$ & DF V \\
\hline D. rerio & 273 & MFEYFRRDT EK R & DF V S A GA A \\
\hline . melanogast & 312 & IFKAFRDDHEKR & DF VLGGGA \\
\hline & 269 & LFREFRNDREKR & DF V S A \\
\hline tropricum & 278 & IFE YFRRDT E KR $R$ & DF V S A GA A \\
\hline
\end{tabular}

Figure 6. Multiple alignment of the amino acid sequence of $C L C N 7$. R286 was located at a highly conserved position. $C L C N 7$, chloride voltage-gated channel 7.

thereby suggesting the possibility of digenic inheritance of osteopetrosis. This study also highlighted the importance of next-generation sequencing for comprehensive molecular diagnosis of osteopetrosis. 


\section{Acknowledgements}

Not applicable.

\section{Funding}

The present study was supported by the National Natural Science Foundation of China (grant no. 31501017).

\section{Availability of data and materials}

The dataset used and/or analyzed during the current study are available from the corresponding author on reasonable request.

\section{Authors' contributions}

YY and YZ planned the project, analyzed the whole exome sequencing data and wrote the manuscript. YY and LL conceived and designed the study. WY, JG, LZ, MT and YY carried out sample collection, clinical diagnosis, Sanger sequencing and microsatellite marker mapping. All authors critically revised, reviewed and gave final approval of this manuscript.

\section{Ethics approval and consent to participate}

The present study was approved by the Ethics Committee of The Hunan Children's Hospital (Changsha, China). Prior to participating in the study, all subjects and guardians of children provided written informed consent.

\section{Patient consent for publication}

Informed consent was obtained from all subjects or their guardians for the publication of patient data.

\section{Competing interests}

The authors declare that they have no competing interests.

\section{References}

1. Sobacchi C, Schulz A, Coxon FP, Villa A and Helfrich MH: Osteopetrosis: Genetics, treatment and new insights into osteoclast function. Nat Rev Endocrinol 9: 522-536, 2013.

2. Stark Z and Savarirayan R: Osteopetrosis. Orphanet J Rare Dis 4: 5, 2009.

3. Deng H, He D, Rong P, Xu H, Yuan L, Li L, Lu Q and Guo Y: Novel CLCN7 mutation identified in a Han Chinese family with autosomal dominant osteopetrosis-2. Mol Pain 12: $1744806916652628,2016$.

4. Palagano E, Blair HC, Pangrazio A, Tourkova I, Strina D, Angius A, Cuccuru G, Oppo M, Uva P, Van Hul W, et al: Buried in the middle but guilty: Intronic mutations in the TCIRG1 gene cause human autosomal recessive osteopetrosis. J Bone Miner Res 30: 1814-1821, 2015.

5. Del Fattore A, Cappariello A and Teti A: Genetics, pathogenesis and complications of osteopetrosis. Bone 42: 19-29, 2008.
6. Waguespack SG, Koller DL, White KE, Fishburn T, Carn G, Buckwalter KA, Johnson M, Kocisko M, Evans WE, Foroud T and Econs MJ: Chloride channel 7 (ClCN7) gene mutations and autosomal dominant osteopetrosis, type II. J Bone Miner Res 18: 1513-1518, 2003

7. Van Wesenbeeck L, Cleiren E, Gram J, Beals RK, Bénichou O, Scopelliti D, Key L, Renton T, Bartels C, Gong Y, et al: Six novel missense mutations in the LDL receptor-related protein 5 (LRP5) gene in different conditions with an increased bone density. Am J Hum Genet 272: 763-771, 2003.

8. Kornak U, Kasper D, Bösl MR, Kaiser E, Schweizer M, Schulz A, Friedrich W, Delling G and Jentsch TJ: Loss of the ClC-7 chloride channel leads to osteopetrosis in mice and man. Cell 104: 205-215, 2001.

9. Pangrazio A, Pusch M, Caldana E, Frattini A, Lanino E, Tamhankar PM, Phadke S, Lopez AG, Orchard P, Mihci E, et al: Molecular and clinical heterogeneity in CLCN7-dependent osteopetrosis: Report of 20 novel mutations. Hum Mutat 31: E1071-E1080, 2010.

10. Balemans W, Van Wesenbeeck L and Van Hul W: A clinical and molecular overview of the human osteopetroses. Calcif Tissue Int 77: 263-274, 2005.

11. Del Fattore A, Peruzzi B, Rucci N, Recchia I, Cappariello A, Longo M, Fortunati D, Ballanti P, Iacobini M, Luciani M, et al: Clinical, genetic, and cellular analysis of 49 osteopetrotic patients: Implications for diagnosis and treatment. J Med Genet 43: 315-325, 2006.

12. Cleiren E, Bénichou O, Van Hul E, Gram J, Bollerslev J, Singer FR, Beaverson K, Aledo A, Whyte MP, Yoneyama T, et al: Albers-Schönberg disease (autosomal dominant osteopetrosis, type II) results from mutations in the $\mathrm{ClCN} 7$ chloride channel gene. Hum Mol Genet 10: 2861-2867, 2001.

13. Lam CW, Tong SF, Wong K, Luo YF, Tang HY, Ha SY and Chan MH: DNA-based diagnosis of malignant osteopetrosis by whole-genome scan using a single-nucleotide polymorphism microarray: Standardization of molecular investigations of genetic diseases due to consanguinity. J Hum Genet 52: 98-101, 2007.

14. Mazzolari E, Forino C, Razza A, Porta F, Villa A and Notarangelo LD: A single-center experience in 20 patients with infantile malignant osteopetrosis. Am J Hematol 84: 473-479, 2009.

15. Li N, Yang Y, Bu J, Zhao C, Lu S, Zhao J, Yan L, Cui L, Zheng R, Li J, et al: An autosomal dominant progressive congenital zonular nuclear cataract linked to chromosome 20p12.2-p11.23. Mol Vis 12: 1506-1510, 2006.

16. Alam I, Gray AK, Chu K, Ichikawa S, Mohammad KS, Capannolo M, Capulli M, Maurizi A, Muraca M, Teti A, et al: Generation of the first autosomal dominant osteopetrosis type II (ADO2) disease models. Bone 59: 66-75, 2014.

17. Coudert AE, de Vernejoul MC, Muraca M and Del Fattore A: Osteopetrosis and its relevance for the discovery of new functions associated with the skeleton. Int J Endocrinol 2015: 372156, 2015.

18. Aggarwal S: Skeletal dysplasias with increased bone density: Evolution of molecular pathogenesis in the last century. Gene 528: 41-45, 2013.

19. Wang C, Zhang H, He JW, Gu JM, Hu WW, Hu YQ, Li M, Liu YJ, Fu WZ, Yue H, et al: The virulence gene and clinical phenotypes of osteopetrosis in the Chinese population: Six novel mutations of the CLCN7 genein twelve osteopetrosis families.J Bone MinerMetab 30: 338-348, 2012.

20. Chu K, Koller DL, Snyder R, Fishburn T, Lai D, Waguespack SG, Foroud $\mathrm{T}$ and Econs MJ: Analysis of variation in expression of autosomal dominant osteopetrosis type 2: Searching for modifier genes. Bone 37: 655-661, 2005.

21. Frattini A, Pangrazio A, Susani L, Sobacchi C, Mirolo M, Abinun M, Andolina M, Flanagan A, Horwitz EM, Mihci E, et al: Chloride channel $\mathrm{ClCN} 7$ mutations are responsible for severe recessive, dominant, and intermediate osteopetrosis. J Bone Miner Res 18: 1740-1747, 2003

22. Yu T, Yu Y, Wang J, Yin L, Zhou Y, Ying D, Huang R, Chen H, Wu S, Shen Y, et al: Identification of TCIRG1 and CLCN7 gene mutations in a patient with autosomal recessive osteopetrosis. Mol Med Rep 9: 1191-1196, 2014. 